\title{
Latest Trends in Sport Equipment Realization Case History Presentation
}

\author{
Emidio M. CENNERILLI*, Serena SANTORO \\ EGS, San Lazzaro di Savena (BO), Italy \\ http://dx.doi.org/10.15221/14.076
}

\begin{abstract}
This article aims at discuss the use of 3D technologies in the manufacturing industry. In particular, it explores how such technologies can benefit to traditional measurement, as well as to the surface modelling of products. The article provides an overview about 3D scanning technologies and reverse engineering software, focusing the attention towards the manner in which complicated and detailed elements can be digitally recorded in a rapid way, to provide a permanent acquisition of dimensions and forms. Furthermore, it will show how the data set produced through the use of a 3D scanner can be elaborated to create a 3D surface mesh.

In particular, this work will describe a real application case about how the use of 3D scanner integrated with computer aided design (CAD) skills can support manufacturers in building customized equipment for the sporting environment: carbon fiber shin guard for football players.

The article concludes that 3D scanners in the manufacturing industry facilitate the collection of highly accurate information in a very short time and allow to develop perfectly fitting, custom and highly performing equipment for sport.
\end{abstract}

Keywords: 3D body scanner, 3D technology, 3D software, CAD/CAM, reverse engineering, customized sport equipment, manufacturing industry, sport goods.

\section{Introduction}

The first industrial revolution began in Britain in the late 18th century, with the mechanization of the textile industry. Tasks previously done laboriously by hand in hundreds of weavers' cottages were brought together in a single cotton mill, and the factory was born. The second industrial revolution came in the early 20th century, when Henry Ford mastered the moving assembly line and ushered in the age of mass production. [1]

Now a third revolution is under way. Manufacturing is going digital and will transform the way goods are made. Indeed, nowadays the manufacturing industry does not just design products in digital but moves a large part of the manufacturing process to digital thanks to the use of $3 \mathrm{D}$ technology.

This paper concerns the application of 3D technologies in the manufacturing industry; by presenting a real application case, the aim of the work is to show all the benefits arising from the use of new production processes. In particular, the case is about an application in a special area, which requires perfectly fitting equipment to gain competitive advantage: the sport.

By focusing the attention on 3D body scanning technologies, the entire process of carbon fiber shin guard creation will be presented - from the leg scanning to the mesh elaboration and product realization.

\section{Scanning technologies overview}

There are several ways to obtain a 3D scanning of physical objects. All of them require to gain information on the spatial position of the surface points of the object. This can be achieved either by physically touching the object with a sensor or by using sound waves or light.

Light in particular is very often used because it is accurate, fast, reliable and non-invasive. Indeed, a touching sensor can damage the object or get damaged during the scanning process.

\subsection{Light-based scanning technologies}

Light-based scanning technologies for short-distance scanning are mostly based on two main categories: laser light and structured light.

\footnotetext{
* egs-info@egsolutions.com; +39 (0)51 6049700; www.egsolutions.com
} 


\subsubsection{Laser light $3 D$ scanner}

In the laser light systems, a laser beam or laser stripe is directed onto the target, where it will be deflected according to the characteristics of the target surface. One or more sensors will then intercept the reflected beam. From the relative position between the light source and reflected beam, the position, the distance and the orientation of the target surface can be mathematically calculated.

Laser-based scanners have a high and vastly adjustable accuracy, but the time required to complete a scan is strongly and directly dependent on the quality expected. Laser scanners acquire data point by point, so for any increase of the amount of points scanned there is a proportional increase in the amount of scanning time required. For extremely detailed scan, the acquisition time can be so long as to generate motion related problems if the object or the scanner moves or are displaced even slightly during the scan. Moreover, laser, unlike structured light, can be dangerous and might require special precautions during the use.

For these reasons, to realize the shin guard has been chosen to proceed with a structured light scanner.

\subsubsection{Structured light $3 D$ scanner}

Structured light systems work with standard light instead of lasers, and project a stable and defined series of light patterns on the target object. The distortion of the reflected image is then captured by cameras, and the position of every point on the target surface is calculated from the distortions of the patterns.

Structured light require multiple scans to thoroughly acquire every surface of the target object. The different scans are then merged together into a single model. Despite the multiple scans required, structured light scanners are not slow. This is possible because the CCD sensors in a structured light scanner can acquire information on multiple points in the field of view at once, instead of just one at a time.

As a final result, the structured light technology is very fast and effective to scan low curves surfaces, and highly detailed organic surfaces.

\section{Manufacturing in sporting environment}

Olympic teams and professional players in general often use the study of athletic body parts to gain competitive advantage. Indeed, having custom-fitted sport equipment makes a big difference in two key areas of advantage: performance and comfort.

Past methods of fit out have been laborious and accurate results was not easily reachable due to long and boring process of trials with material wasting before getting right.

Today, the use of 3D technologies together with sophisticated materials, allows players to wear equipment that can be considered real extensions of the body. Consequently, the movements are not influenced by other elements but are totally natural.

At the same time, the use of 3D scanners improves the works of manufacturers because by digitalizing the entire process companies can reduce production process's time and costs. Indeed, getting digital data requires less time and generates repeatable results without material wasting.

Furthermore, the work is more accurate and significantly simplified under many points of view. This is confirmed by Enrico Campari, General Manager at the "Campari Compositi Per Campioni"". The plaster cast technique forced him to travel around the world with heavy and fragile plaster casts, but now with just a suitcase and a USB he can establishes a global presence in foreign markets.

\section{Case history}

On the occasion of the Football European Championship hosted by Poland and Ukraine in 2012, the legs of national players from the most important football clubs have been scanned to create new, sophisticated and better fit carbon fiber shin guards.

The project has been developed by two companies, "EGS" (technical partner) and "Campari Compositi Per Campioni" (manufacturer partner).

EGS is a company with more than 15 years of experience in the CAD/CAM Industry. It is recognized for its leading knowhow in the production and offer of 3D innovative solutions, among with scanners, reverse engineering software and CAD systems.

\footnotetext{
Company description in the following chapter.
} 
On the other hand, Campari Compositi Per Campioni is a manufacturing company which designs and manufactures protection in carbon fiber, kevlar, zylon and aeronautic glass, since 1994. In particular, it is specialized in creating anatomical protections for the human body, especially for professional players.

The main goal of the collaboration has been to develop a reliable and repeatable series of digital procedures by bringing innovation in a segment that had not evolved for lack of research.

\subsection{The scanner}

For this project has been used the Artec EVA structured light scanner (see figure nr. 1 below). It is an handle scanner designed for accurate, close-in work. It is good for organic shapes acquisition, such as human bodies, because can generates industrial accuracy level results. The 3D scanner Artec EVA combines a projected light and cameras system. By projecting a band of light on a surface, the band suffers distortion according to the surface. The two cameras capture this distortion and reconstruct the exact geometric shape of the element, by creating a cloud of points. This process allows to have a perfect three-dimensional measuring of the element.

The use of this technology is becoming so widespread that nowadays, in order to facilitate the work even to non-experts of technology, there are auto-rotating platforms that further simplify the scanning phase.

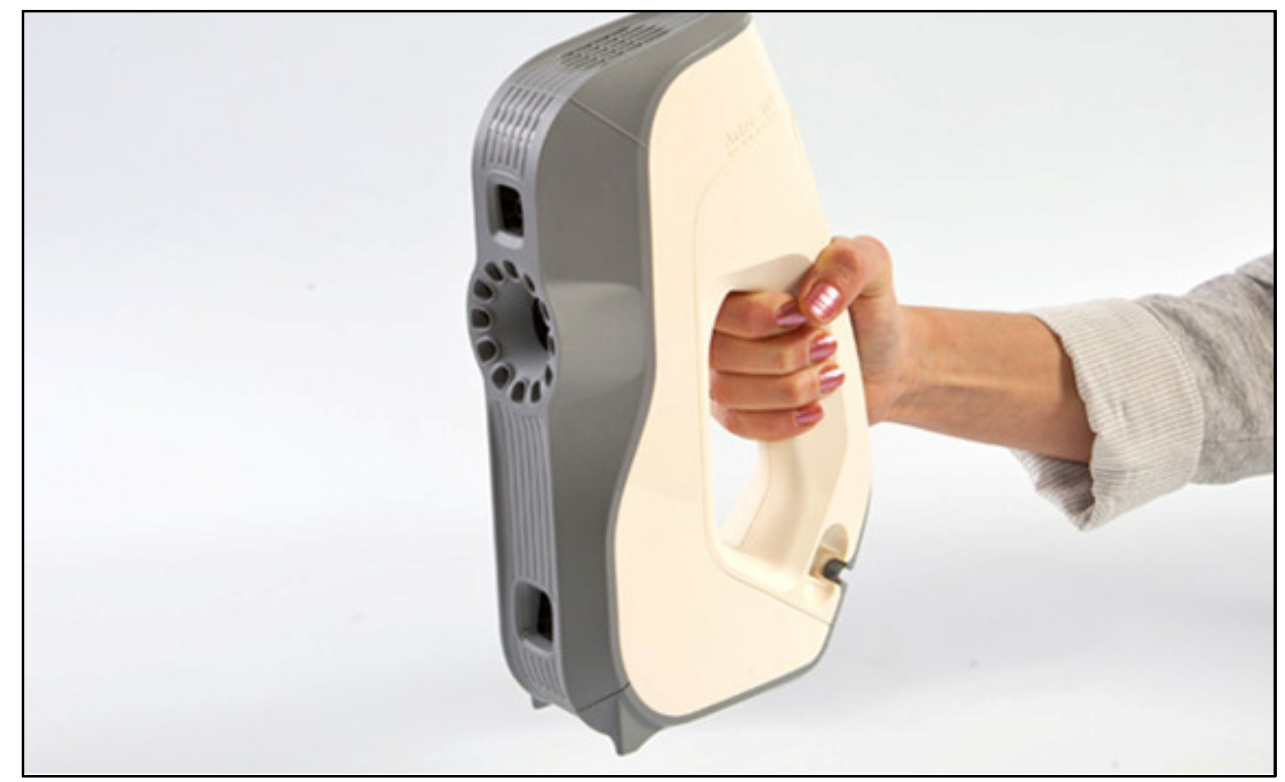

Fig. 1. Artec EVA - structured light scanner.

\subsection{The scanning phase}

The first step of the project was to accurately scan the athletes with a 3D handle scanner for capturing all surface's details required for each customization.

This procedure took just few minutes for getting the 3D image because the Artec EVA is able to captures up to 16 frames per second, which are automatically aligned in real-time. Indeed, just by moving the scanner around the leg of the athlete, the real-time surface alignment gives a good understanding of what has been scanned so far and what still needs scanning.

Two scans have been made to capture the whole element: the first from one side of the leg and another one from the opposite side (see figure $\mathrm{nr} .2$ below).

Comparing to the past, today the use of $3 \mathrm{D}$ scanner improves the work with several benefits. On the player's side, the shin guard's quality have been improved: previously they were heavy and bulky to carry on, now they are lighter and perfectly fitting.

On the manufacturer side, the production process is easier and more accurate. The plaster cast technique was a long, laborious manual procedure of trials before getting right. Moreover, another important difference is the fact that with the use of the scanner, no errors can occurs and no material is wasted (since it is not necessary to use plaster). All the information (measurements, surface details and texture) are saved in a single file, which can be easily modeled and transferred. 


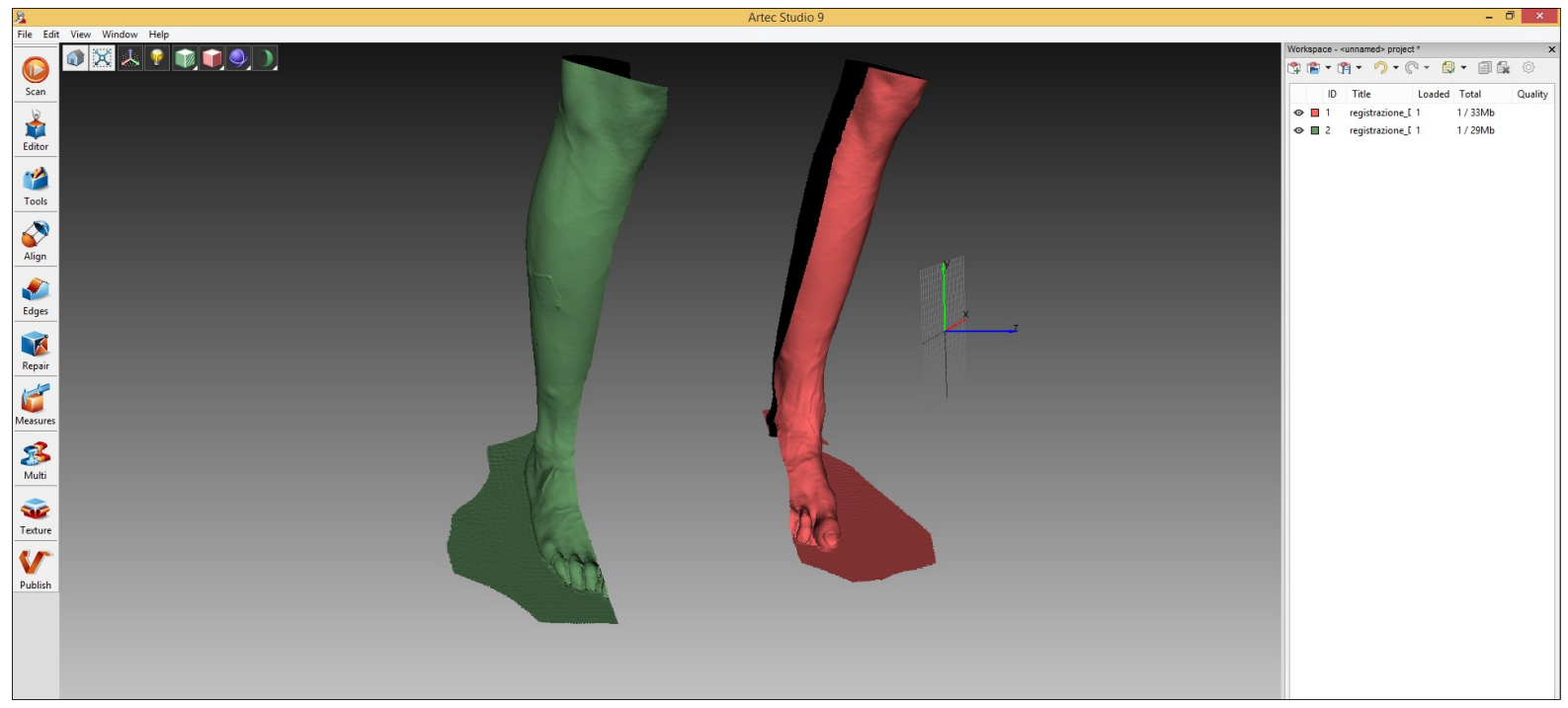

Fig. 2. $3 D$ file of the two scans.

\subsection{Scans alignment, fusion and mesh export}

After the scanning step, the two (ore more) scans must be aligned together in order to get the full element (in this specific case, the 3D leg of the athlete). During this step we pass from the point cloud to the mesh. In order to do this procedure has been used the 3D software of the scanner (Artec Sudio). It is a very short and easy phase because the automatic optimizations algorithm of this software can align and fuse the scans very quickly.

First of all, in the alignment (or registration) procedure, for the user has been sufficient to follow the "3 points rule". According to this method, the scans can be aligned just by assigning 3 points on the first scan (if points are far from each other, the merger will be more accurate). Following, other 3 points have been identified on the second scan, in a position similar to those on the first scan. In this way, the software is able recognize the common surfaces and to merge them together. This procedure can be repeated for all the scans made. It is possible to see if the procedure has been successfully completed by looking at the color of the scan. Indeed, as shown in the picture below (see figure nr. 3 below), each scan has got a specific color and the fusion can be considered good when the color is a perfect mix of the two single scans.

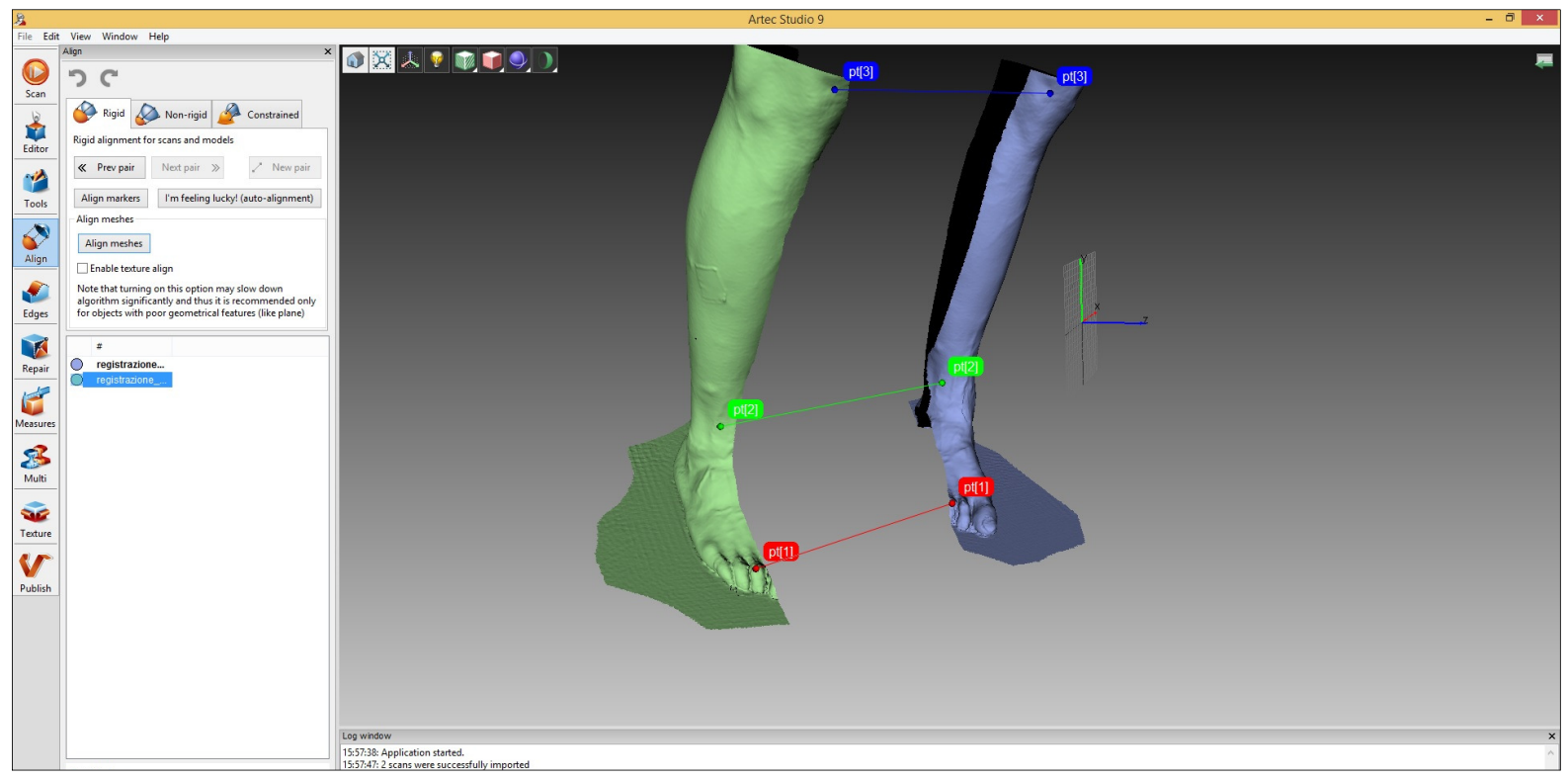

Fig. 3. Artec Studio: scans alignment with the automatic optimizations algorithm. 
As a result, the point cloud of each scan was combined in a single "puzzle" with the other, so that the entire 3D picture was correctly positioned in space. In case you need to work the points cloud before the fusion phase, Artec Studio has got a command for the points cloud optimization (noise filter, handling outliers, etc.) that, thanks to automatic algorithms, allow also to not-expert users to complete the work in the best way.

It is important to underline that, contrarily to the traditional analogic technique, the digital process is not unidirectional: if something is missing, it is possible to go back to the scan step and acquire just the necessary part.

After completing and optimizing the registration phase, it is necessary to transform the points cloud in a mesh (.stl file). This is called "fusion process" and means that all points are merged into triangles according to a certain order. Also in this case, the automatic algorithms of the system can optimize the final structure of the mesh. In particular, for the fusion of the leg scans has been chosen to proceed with a type of fusion called "smooth" because it is able to compensate for any movements made by the subject during the scanning. For other cases, such as inanimate objects or complex geometries, Artec Studio provides two other different fusion strategies: "fast" or "sharp" (see figure nr. 4 below).

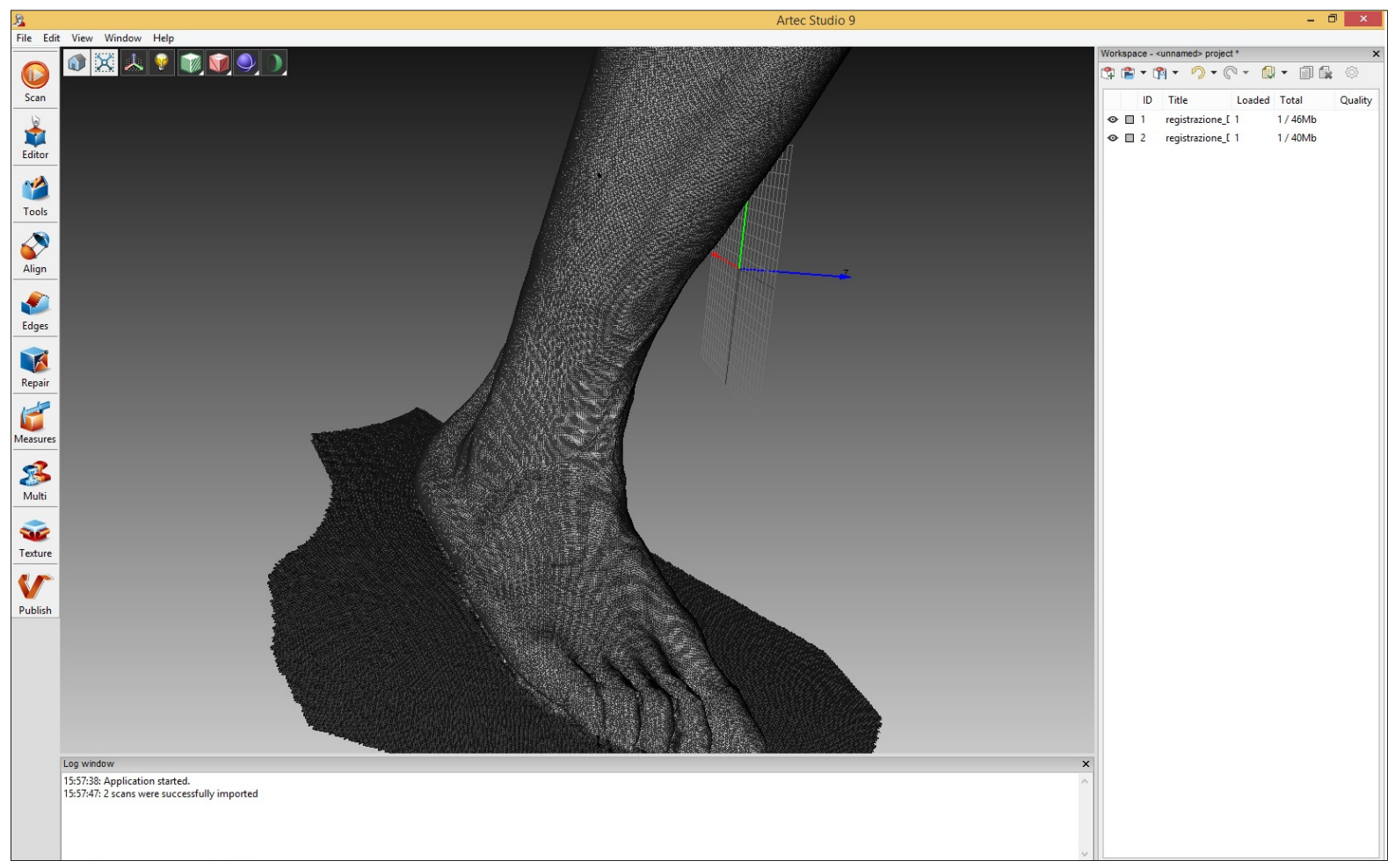

Fig. 4. Artec Studio: fusion process (mesh).

\subsection{The modeling}

At this point, the resulting dataset of the scanning process is a three-dimensional picture (mesh) workable with reverse engineering software. In particular, has been used Leios, a powerful instrument for the 3D data elaboration. It is important to say that this tool has a direct integration with Artec Studio and, therefore, it is very easy continuing the work in a direct way through the command "export in Leios".

Through the use of Leios, was possible to make editing of triangle meshes and, more specifically, to realize direct sculpting on meshes for prototyping.

Once in the system, boolean operations on the data have been used to enable precise modeling of the fit-out, customized perfectly to the athlete's unique body part shaped. Advanced algorithms quickly process the captured information and enabled users to export finalized data in a variety of common file formats.

Several strategies can be used inside Leios to optimize a model starting from the scanned dataset. The user can choose the most useful strategy according to his/her needs and to his/her field of work. In addition, strategies can be combined to achieve the desired result.

In this specific case, was useful to map the areas of excessive heat and sweat in order to design more comfortable fabrics. 
Moreover, thanks to the user-friendly interface, was easy to close the holes by using the "fill-hole" tool (see figure nr. 5 and 6 below) and the different types of hole selection (total, partial or bridge) and filling ("flat" or "with curvature").

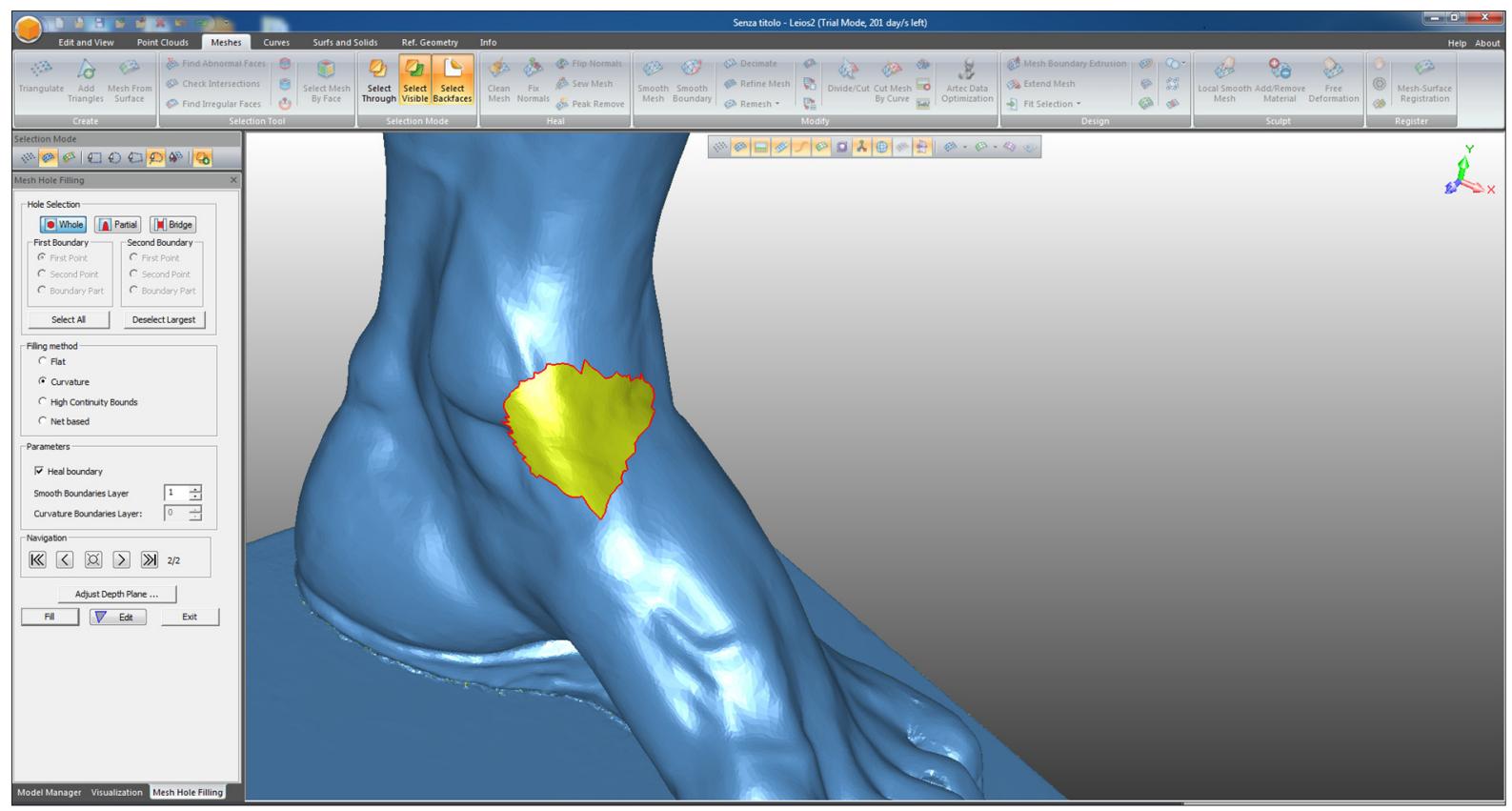

Fig. 5. Leios: hole selection.

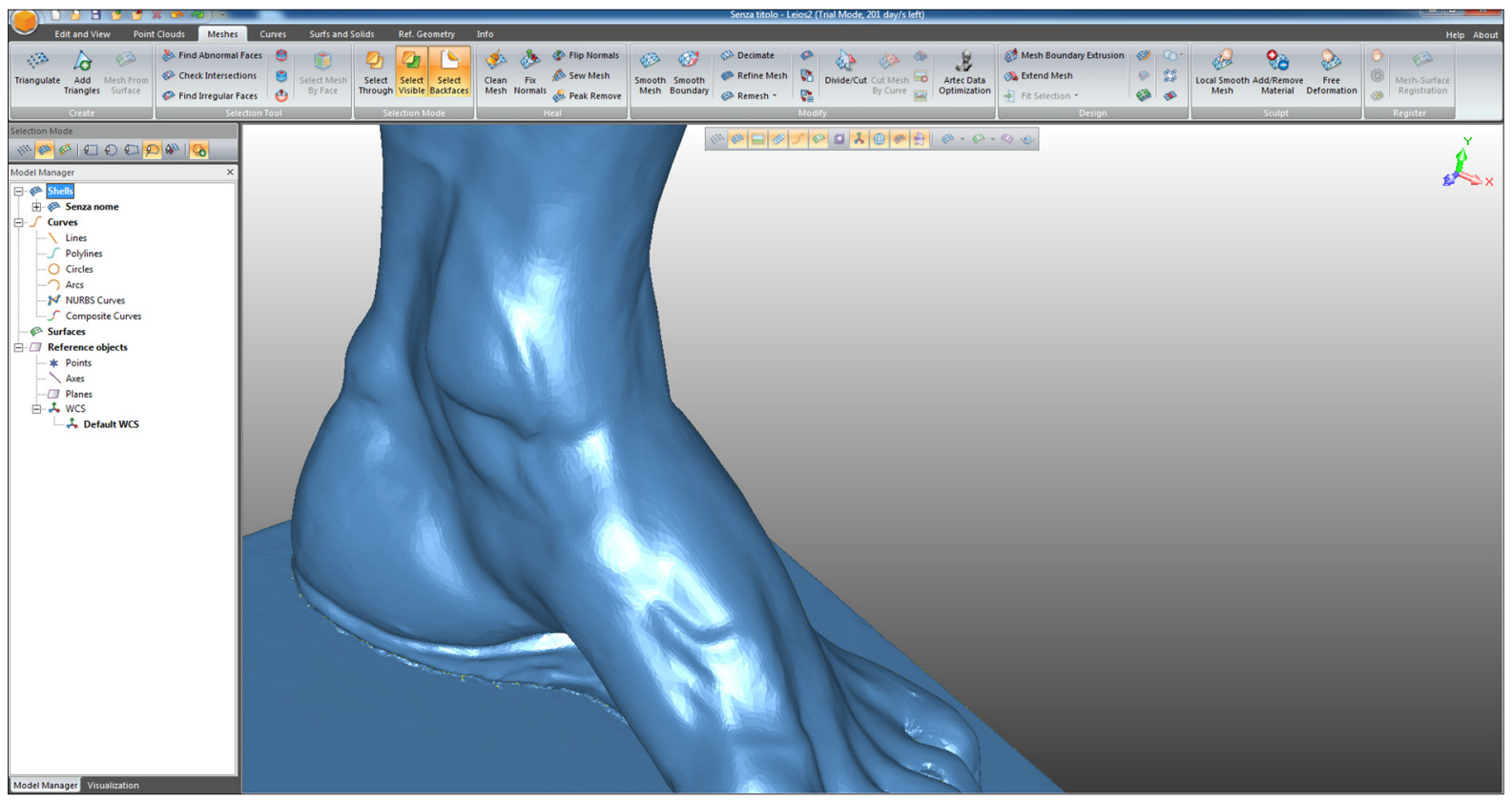

Fig. 6. Leios: fill hole.

Subsequently, has been made the edge recognition and analysis to dissect automatically the mesh in several regions divided by edges and was controlled each step by analyzing the results with powerful inspection tools.

Moreover, for the realization of the customized shin guards, has been made a mesh boundary extrusion (see figure $\mathrm{nr} .7$ below) in order to add the perfect offset of $3 \mathrm{~mm}$ for the terry cloth padding. A peculiarity that distinguishes the software Leios, is the very high quality of the mesh which is able to work. In fact, after the optimization processing described, the mesh was ready for the 3D printing. 


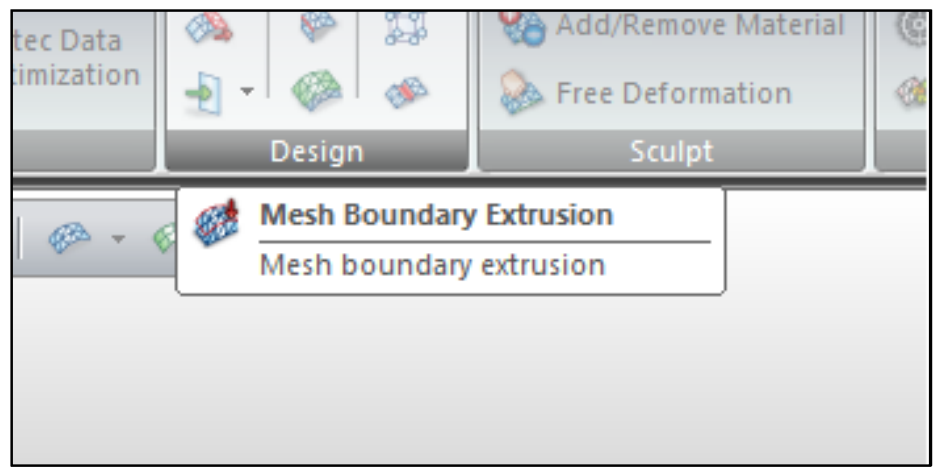

Fig. 7. Leios: mesh boundary extrusion function.

Finally, Leios features powerful elaboration and editing tools allows to do auto-surfacing or manual patching to create NURBS mathematical model on the mesh in order to exports the datasets in CAD format to make any changes or customizations to the product.

\subsection{The final realization}

After the modeling step, the final realization of the product goes through the 3D printing process. Indeed, the optimized 3D image of the player leg worked with Leios is printed out in a special material, which can resist to very high temperatures: the nylon aluminum.

The Campari Compositi Per Campioni has realized the shin guards in carbon fiber, a material widely used in the automotive industry but still little known in football. It guarantees lightness, overcoming the athlete hostility towards disturbing protections; at the same time, it is as strong as steel: the perfect combination that allows the creation of a world class product.

The use of the nylon aluminum for the leg rapid prototyping allows to work the carbon fiber in the autoclave (carbon fiber polymerization process) in order to obtain very accurate results.

According to this process, the carbon fiber is treated in the autoclave at 120 Celsius degrees. The polymerization process makes sure that the carbon fiber over the leg model is deformed according to the anatomy of it, ensuring a perfect adjacency on every single point.

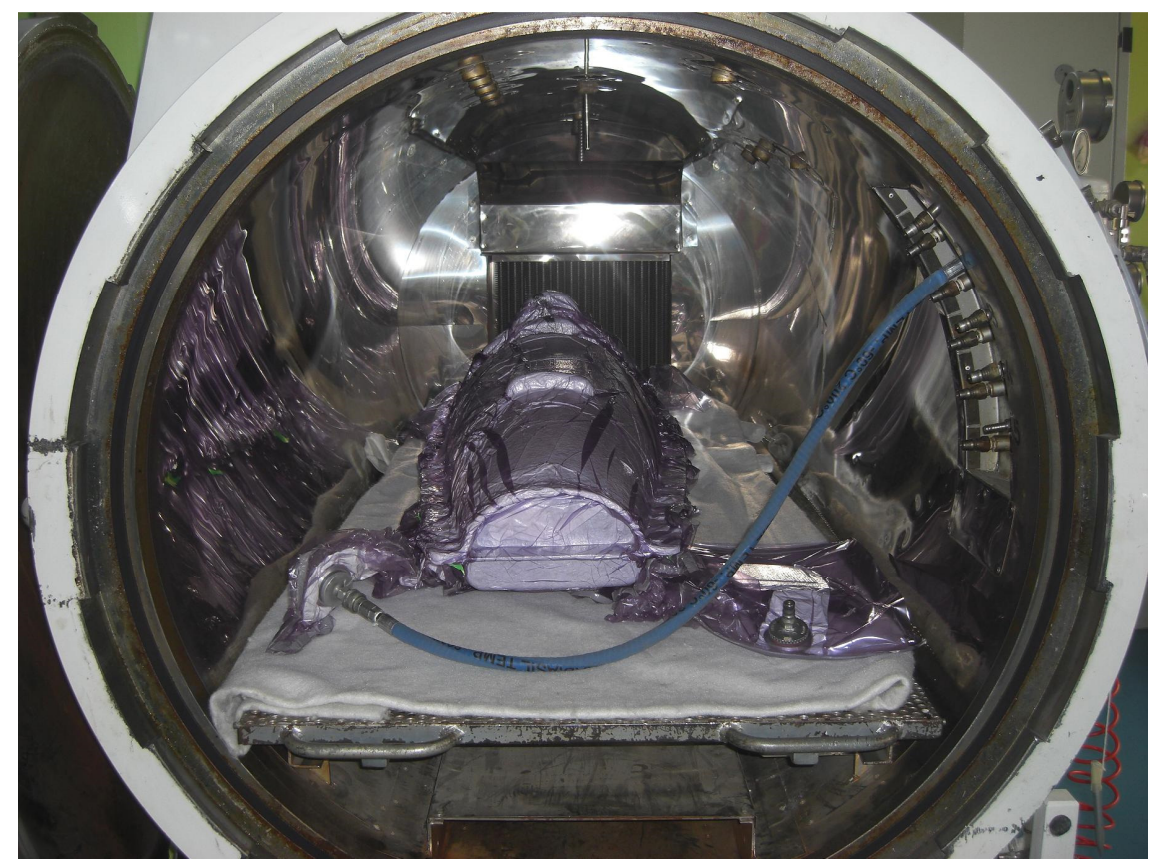

Fig. 8. Autoclave for the carbon fiber polymerization process.

The entire process, from the scanning phase to the rapid prototyping and polymerization, allows to earn a lot of time that could be used for the improvement of the final confection manufacturing activity. 


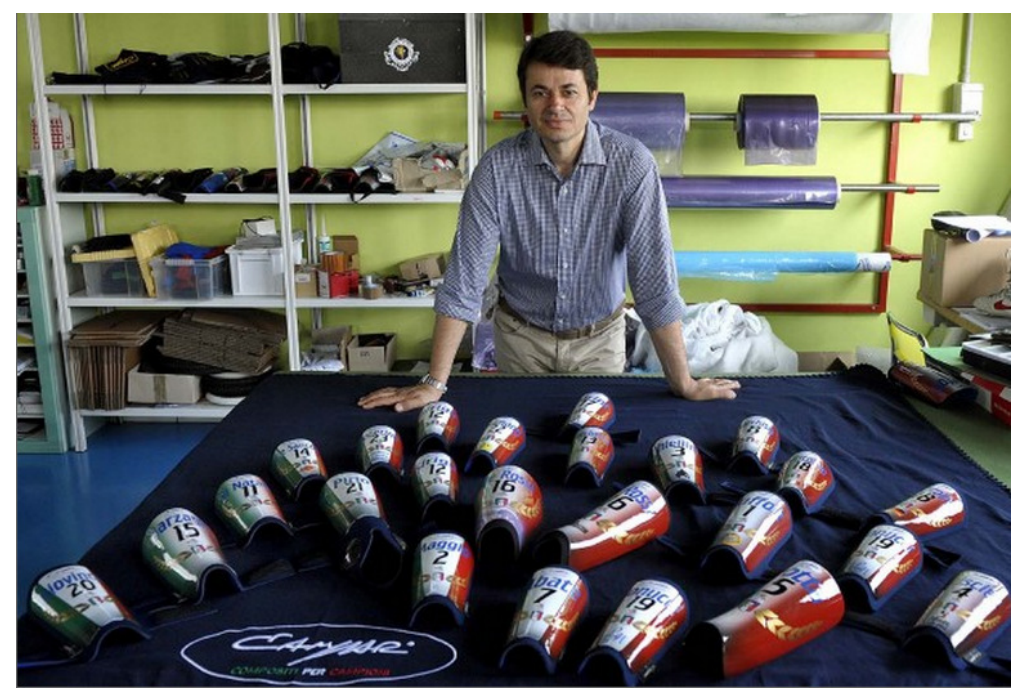

Fig. 9. Enrico Campari, General Manager at Compositi Per Campioni S.a.s.

\section{Conclusion}

With past methods, the process for getting perfect fit-out was long and laborious, characterized by several trial, errors and material wasting. Moreover, the results was not easily repeatable.

The integration of 3D body scanner in the manufacturing industry gives the opportunity to improve the workflow in a digital way, by replacing the plaster cast technique, which forced manufacturers to travel around the world with delicate and bulky instruments. Indeed, the case presented shows that thanks to the use of the 3D scanner Artec EVA, which can capture in detail the leg of the player, the sport equipment realized have an exact fitting with an unmatched quality and comfort.

In addition, the new applications with multi-material 3D printing and the opportunity to store the data acquired with the body scanner, make each project repeatable in a very short time - by enhancing this business in a new potential level.

This paper has presented a specific application case in football, anyway, the procedures described can be applied in many other cases such as for anatomical protections for any part of the human body or for the creation of perfectly fitting clothing and sports instruments and prosthesis (see figure nr. 10 below).

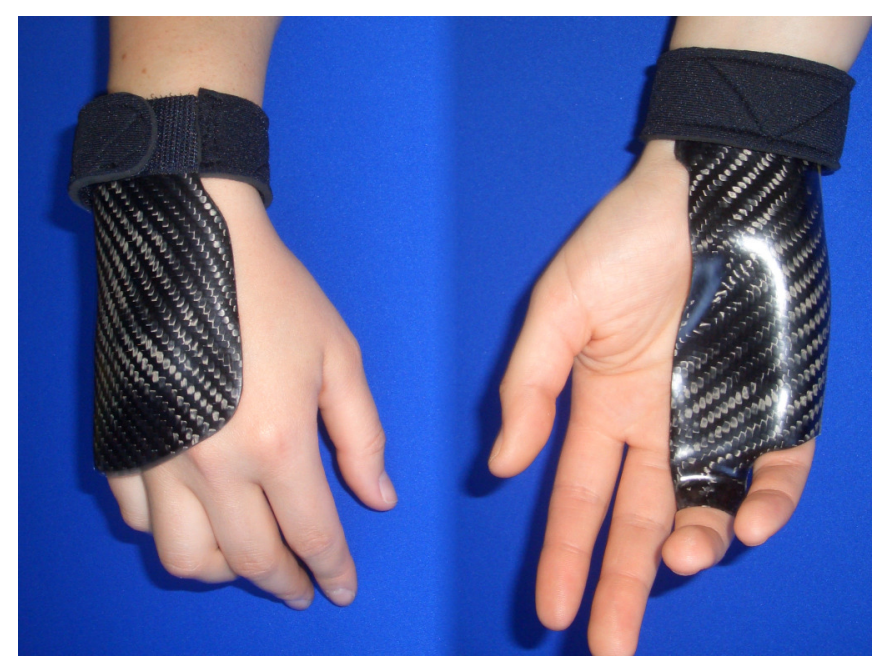

Fig. 10 Customized hand prosthesis in carbon fiber.

\section{References}

[1] The third industrial revolution, http://www.economist.com/node/21553017, accessed 2014. 